\title{
Determining deviations of output variables from forecast values in system for monitoring external round grinding process
}

\author{
Elmar Yagyaev ${ }^{1, *}$ \\ ${ }^{1}$ Crimean Engineering and Pedagogical University, Uchebniy side st.8, 295055 Simferopol, Russian \\ Federation
}

\begin{abstract}
The article considers the estimability of the deviations of actual output variable values from the forecast ones in the system for monitoring the process of external round grinding. The author proposes the method for determining the deviations and establishing their most probable values as well as for establishing the cause of such output variable value deviations and technological system changes. It is substantiated that the determination of the output variable deviations and the parameters of the technological system state is possible at the control of the output variable change rate as the interruption time intervals are different for different subsystems. The authors managed to determine the dependences of the deviations of actual values $(t)$ from the estimated ones $y_{p}(t)$. To resolve the objective of identifying the causes of output value deviations from the forecast ones, the authors applied the method for determining the indirect measurement errors. The obtained dependences made the authors draw the conclusion that at the control of the change rate of output variable values one is able to find out the disturbance type and the type of the deviation of output variable actual values in the technical system. This provides for the opportunity to correct the control of the external round grinding process as well as to calculate the time intervals at which one needs to take into account some or other changes in the technological system.
\end{abstract}

\section{Problem statement}

The peculiarity of contemporary machinery manufacture causes the increase of the requirements to the quality and precision of treated items. The quality of ready products, in its turn, is determined by an industrial process which shall have a high reliability degree and guarantee the parameters of manufactured parts.

One of the areas of the part quality provision at external round grinding is the application of a system for monitoring the technological equipment and cutting.

Multiple methods for cutting diagnostics and control have been developed and put into operation. One of the first researchers to offer the system of diagnostics and adaptive control was B.S. Balakshin [1]. They were further developed in the research [2-11].

\footnotetext{
* Corresponding author: elmar1875@gmail.com
} 
However, existing diagnostics and control methods do not fully take into account the peculiarities of the industrial grinding process.

The grinding diagnostics methods applied at the production site are based on the following:

1) evaluation of the parameters of the technological system state involving complex diagnostic equipment during equipment installation;

2) analysis of the results of the quality parameters' measurement conducted for the treated surfaces and a range of cutting parameters (cutting forces, acoustic emission, etc.).

Both the first and the second options are hardly applicable in the automated production conditions as they do not allow one to identify the changed parameter of a technological system in an unambiguous way, let alone to forecast the system behaviour at the further operation.

In the process of cutting not only the treated part parameters change but also the ones of a technological system. The change in the subsystem parameters causes the change in output variables $y(t)$, for example, the rate of material removal $\Delta r$ and the surface roughness $R a$ by the value $\Delta y$.

The paper purpose - to resolve the problem of identifying the causes inducing the deviations of output variables from the forecast values within the system of monitoring which operates on the dynamics of output variable changes.

\section{Materials and methods of research}

In compliance with the functional monitoring system circuit developed in the paper [12] for external round grinding (Fig. 1), the key tasks are the analysis of the dynamics of the output variable change and the parameters of the technological system state.

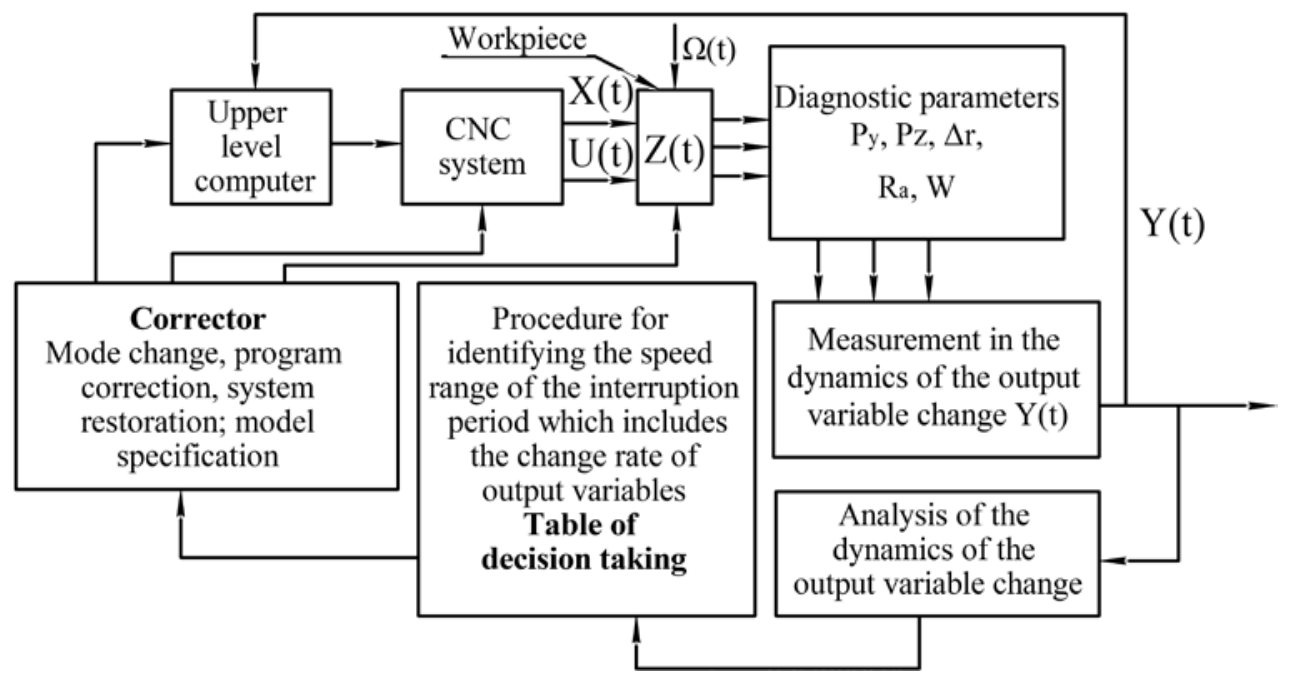

Fig. 1. Generalized functional scheme of the machine diagnostics\&control system with the grinding cycle correction.

At grinding, after the interruption, the technological system or its subsystem parameters are partially or fully restored (workpiece replacement, circle correction, tool/device replacement). As a result, output values $y(t)$ (material removal rate after circle correction $\Delta r_{b}$ changes by the value $\Delta\left(\Delta r_{6}\right.$ at the next cycle), material roughness change by the value $\Delta R a_{6}$ at the beginning of the next cycle. 
The conducted analysis and the synthesis of grinding machine operation practice make the authors draw a conclusion that the control of output variables $y(t)$ at the moment of time $t$ does not allow for the determination of the technological system state parameters the measurement of which caused output value deviations. Such possibility arises at the control of the change rate of output variable values $y(t)$ as the interruption time intervals $T$ for various subsystems significantly differ.

In the paper [13] the authors simulated the state space with the consideration of the possibility to restore system parameters for the grinding process. As a result, we obtained the following equations:

$$
\begin{gathered}
z_{i, j}(t)=z_{i j-1}+A_{i j}(j-1)+A_{z i j}\left(t-\sum_{k=1}^{j-1} t_{u k}\right), \\
y_{i}(t)=D_{i}(t) z_{i}(t) ; \\
\dot{y}_{i}(t)=D_{i} \dot{z}(t) .
\end{gathered}
$$

where $z(t)$ - vector of the technological system state parameters; $U(t)$ - command input vector; $A(t), B(t), D(t)$ - matrices of corresponding factors; $A_{i j}$ - operator taking into account the restoring of the $i$-th system parameter after the execution of the $(j-1)$-th cycle (before the start of the $j$-th cycle); $A_{z i j}$ - operator defining the change in the system parameter at the operation in the $j$-th cycle; $\tau_{\text {LK }}$ - time of the $k$-th cycle execution.

In addition, the system is completed with the procedure for defining the speed range $y_{1}(t), y_{2}(t), \ldots y_{m}(t)$ of one or another interruption period the output variable change rate included into:

$$
\dot{y}_{i}(t) \in\left[\begin{array}{c}
\dot{y}_{1}(t) \\
\dot{y}_{2}(t) \\
\ldots \\
\dot{y}_{m}(t)
\end{array}\right] .
$$

Considering the obtained dependences(1)...(4), one can make a conclusion that, in case the changed parameter of the technological system state is defined by the equation of the choice of the nearest values from the set (4), therefore, obtaining the information on the output variable value at any time moment, one can define the parameter value.

The evaluation of deviations of actual output variable values from the forecast ones includes two key stages:

1) identification of deviations and finding out their most probable values;

2) establishing the causes of deviations.

We further consider the set of tasks to resolve these stages for the analyzed control process. Let the deviations of actual values $y(t)$ from the estimated ones $y_{p}(t)$ be defined by the dependence

$$
\Delta y(t)=y_{p}(t)-y(t)
$$

If the output variable measurement is frequently conducted within the limited time interval at $\varphi(t)=1(t)$ and one does not register any significant changes of $y(t)$ during this period, the presence of deviations can be defined with any confidence level by means of the methods of the sample observations' analysis. At the sampling average values comparison the authors made a null hypothesis on the equality of general average values. It is rejected in case 


$$
\left|\bar{y}_{1}-\bar{y}_{2}\right| \geq t_{1-\frac{\alpha}{2}} \cdot S \sqrt{\frac{1}{n_{1}}+\frac{1}{n_{2}}}
$$

where $\bar{y}_{1}$ and $\bar{y}_{2}$-comparable sampling average values; $n_{1}$ and $n_{2}$ - scope of the first and second sampling; $t_{1-\alpha / 2}$ - Student criterion, defined with the confidence level $1-\alpha / 2$ and the number of the degrees of freedom $f=n_{1}+n_{2}-2 ; S^{2}$ calculated by the sample variances

$$
S^{2}=\frac{\left(n_{1}-1\right) S_{1}^{2}+\left(n_{2}-1\right) S_{2}^{2}}{n_{1}+n_{2}-2} .
$$

For the considered process the subset of estimated values serves as the first sampling while the subset of output variable measured values serves as the second sampling. Accordingly, $S_{l}$ and $S_{2}$ are considered as the dispersions of forecast and actual values $y$. At the set conditions $n_{1}=n_{2}$ the inequality (6) takes the form

$$
\left|\bar{y}_{1}(t)-\bar{y}_{2}(t)\right| \geq t_{1-\frac{\alpha}{2}} \sqrt{\frac{S_{1}^{2}+S_{2}^{2}}{n}}
$$

At the dispersion comparison, the authors made a null hypothesis on the equality of general dispersions. The hypothesis is rejected if the Fisher test estimated values are higher than the table ones at the set value of the confidence level. The estimated criterion is defined from the ratio

$$
F=\frac{S_{2}^{2}}{S_{1}^{2}}
$$

If the output variable is not permanent in time but does not have regular highfrequency deviations, within the limited time interval one can also use the dependences (8) and (9) with the preliminary approximation of experimental data (for example, with the help of the polynomial of the $k$-th degree) and the replacement of the left part in the equation (8) by the difference of estimated values within theoretical and empirical dependences. At the calculation of dispersions one should replace $S^{2}$ by the dispersion of adequacy defined from the equation

$$
S_{a d}^{2}=\frac{\sum_{i}\left[y\left(t_{i}\right)-y_{p}\left(t_{i}\right)\right]^{2}}{n-k-1},
$$

where $y\left(t_{\mathrm{i}}\right) \quad$ actual values; $y\left(t_{\mathrm{i}}\right)$ estimated values; $k$ degree of the approximation polynomial.

If the output variable has high-frequency regular modulations, the application of dependences (8) and (9) is impossible as the considered method does not stipulate for the division of deviations into regular and stochastic, and all of them are taken into account at the dispersion calculation. In this cases the analysis requires the application of a more complex mathematical apparatus of stochastic processes.

To simplify the analysis, we proceed to the centered variables decomposing $y(t)$ into a constant component $\mathrm{y}<y(t)>$ and deviation $x(t)$. In this case the dependence (5) has the form

$$
\Delta y(t)=y(t)-<y(t)>-x(t)
$$


In the equation (11) $y\left(t_{i}\right)$ and $<y(t)>$ serve as deterministic functionals, $x(t)$ defines a stochastic process. We present a stochastic process in the form of a stochastic function defined by the Langevin equation [14].

$$
\frac{d}{d t} x(t)+v \cdot x(t)=f(t)
$$

where $f(t)$ - white noise, $v$ - parameter not depending on the coordinate $t$.

The stochastic process $x(t)$ is referred to as the Ornstein-Uhlenbeck process, or the OU process in brief. The key criteria of this process which are the most informative at the set problem resolution are the following:

1. Linear functional

$$
R_{e}=\frac{1}{T} \int_{0}^{T} x(t) d t
$$

2. Deviation mean square functional

$$
R_{q}^{2}=\frac{1}{T} \int_{0}^{T} x^{2}(t) d t
$$

where $T$ - duration of the considered time interval.

To clarify the general method for the definition of the distribution of functionals $R_{e}$ and $R_{q}{ }^{2}$, we consider the Cauchy problem for the OU process assuming the universal reference point $t_{0}=0$. The solution of the equation (12) for these conditions is recorded by a linear functional of white noise $f(t)$

$$
x\left(t ; x_{0} ; 0\right)=x_{0} e^{-v t}+\int_{0}^{t} f(\tau) e^{-v(1-\tau)} d \tau
$$

To obtain the transition density of the probability $W\left(x ; t ; x_{0} ; t_{0}\right)$, we record the OU-process as follows

$$
\mathrm{W}\left(x ; t ; x_{o} ; t_{0}\right)=<\delta\left[x-x\left(t ; x_{0} ; 0\right]>\right.\text {. }
$$

After the replacement of the $\delta$-function with the Fourier integral and transformations for the Gaussian process we obtain

$$
\mathrm{W}\left(x ; t ; x_{o} ; 0\right)=\left[\frac{1}{\pi \sigma_{\mathrm{x}}\left(1-e^{-2 v t}\right)}\right]^{1 / 2} \exp \left[-\frac{1}{\sigma_{x}} \frac{\left(x-x_{0} e^{-v t}\right)^{2}}{1-e^{-2 v t}}\right],
$$

where $\sigma_{x}=\sigma_{f} / v$ - the OU-process intensity.

The obtained function satisfies the Fokker-Planck equation

$$
\frac{\partial}{\partial t} W\left(x ; t ; x_{0} ; 0\right)=v \frac{d}{d x}(x W)+\frac{1}{2} \sigma_{f} \frac{\partial^{2} W}{\partial x^{2}}
$$

with the initial condition

$$
\mathrm{W}\left(x ; 0 ; x_{o} ; 0\right)=\delta\left(x-x_{0}\right) .
$$

As $x(t)$ - Gaussian process, the value $R_{e}$, due to its linearity, also has the Gaussian property and it is sufficient to know the first two moments for its description: 


$$
\begin{gathered}
<R_{e}>=\frac{1}{T} \int_{0}^{T}<x(t)>d t \\
<R_{e}^{2}>=\frac{1}{T^{2}} \int_{0}^{T} \int_{0}^{T}<x(t) x\left(t^{\prime}\right)>d t d t^{\prime}=2 \sigma_{x} \frac{-1+v T+e^{-v T}}{v^{2} T^{2}} .
\end{gathered}
$$

The density of the distribution of the Gaussian stochastic value $Y=R_{e}$ at the known $<R_{e}{ }^{2}>$ is defined as

$$
f_{Y}(y)=\frac{1}{\left(2 \pi<R_{e}^{2}>\right)^{1 / 2}} \exp \left(-\frac{y^{2}}{2<R_{e}^{2}>}\right)
$$

Therefore, the functional $R_{e}$ is Gaussian with the mathematical expectation equal to zero and the dispersion $\left\langle R_{e}{ }^{2}>\right.$.

To evaluate the statistics of the integral quadratic functional, it is convenient to use the apparatus based on the calculation of its generating function

$$
Q(\lambda)=<\exp \left(-\lambda R_{q}^{2}\right)>
$$

where $\lambda$-positive parameter.

The obtained replicating function allows one to define the first and second moments of the stochastic value

$$
\begin{gathered}
<J_{x}>=\frac{1}{2} \sigma_{x} \\
<J_{x}^{2}>=\frac{1}{T^{2}} \iint_{00}^{T T}<x^{2}(t) x^{2}\left(t^{\prime}\right)>d t d t^{\prime}=\left(\frac{1}{2} \sigma_{x}\right)^{2} \frac{-1+2 v T+v^{2} T^{2}+e^{-2 v T}}{v^{2} T^{2}} .
\end{gathered}
$$

To design the distribution density $\rho(\mathrm{J})$, one needs to perform the Laplace numerical procedure of reverse transformation. It is performed in the paper [15].

$$
\begin{aligned}
& p(J)=\sqrt{\frac{T}{2 \pi v \sigma J}} r_{c}\left[\frac{4 v r_{c}}{\left(r_{c}+v\right)^{2}-\left(r_{c}-v\right)^{2} \exp \left(-2 r_{c} T\right)}\right]^{1 / 2} \times \\
& \times \exp \left[-\frac{v T}{2 \sigma_{x}}\left(\sqrt{J}-\frac{\sigma_{x}}{2 \sqrt{J}}\right)^{2}\right]
\end{aligned}
$$

where $r_{c}=\frac{v \sigma_{x}}{2 \eta}$.

Having the data on the statistical characteristics of a stochastic process $x(t)$, we proceed with the corresponding process analysis $\Delta y(t)$. As the components $y(t)$ and $\bar{y}(t)$ serve as determinated functionals, then

$$
\begin{aligned}
& <R_{e \Delta y}>=<\Delta y(t)>=y(t)-<\bar{y}(t)>-<R_{e}>=y(t)-<\bar{y}(t)> \\
& <R_{e \Delta y}^{2}>=<R_{e}^{2}>
\end{aligned}
$$

We shall evaluate the significance of deviations $\Delta y(t)$. To do this, we consider the stochastic value $Y=\bar{y}(t)-y(t)$. The probability of the event $P(Y>0)$ is defined by the distribution density 


$$
P(Y>0)=\int_{-(y(t)-<\bar{y}(t)>)^{+}}^{+\infty} f_{Y}(y) d y
$$

The probability of the event $P(Y<0)$ is correspondingly calculated

$$
P(Y<0)=\int_{(y(t)-<\bar{y}(t)>)^{+}}^{+\infty} f_{Y}(y) d y
$$

Generalizing the dependences (28) and (29) and, denoting the probability of the correctness of the decision on the significance $\Delta y$ as $D_{Y}$, we finally obtain

$$
D_{Y}=\int_{-|y(t)-<\bar{y}(t)>|}^{+\infty} f_{Y}(y) d y
$$

The final solution of the set problem requires one to find the confidence level $P_{D}$ and compare it with the estimated value of the criterion $D_{Y}$.

At the comparison of dispersion values we shall do the same thing. The estimated dispersion is a determinated functional, it has a $\delta$-shaped density of distribution, and the dispersion distribution density of a stochastic process is known (26). The probability of correct decision taking on the dispersion substantial difference will be defined as

$$
D_{S}=\int_{-\left|S-<S_{x}>\right|}^{+\infty} P(y) d y
$$

At the presence of a regular part within a stochastic process one considers the functional of the type

$$
J_{S+x} \equiv R_{S+x}^{2}(T)=\frac{1}{T} \int_{0}^{T}[x(t)+S(t)]^{2} d t
$$

which is divided into three additive components

$$
J_{S+x}=\frac{1}{T} \int_{0}^{T} S^{2}(t) d t+\frac{1}{T} \int_{0}^{T} x^{2}(t) d t+\frac{2}{T} \int_{0}^{T} x(t) S(t) d t
$$

The first additive component of the dependence (33) is not stochastic, the statistics of the second one was previously studied, the analysis of the statistics presented for the third additive component is possible only at the specific regular function setting $S(t)$. The generalized functionals used to conduct this analysis have the following form

$$
\begin{gathered}
<J>=\frac{1}{2} \sigma_{x}+\frac{1}{T} \int_{0}^{T} S^{2}(t) d t \\
<J^{2}>=\left(\frac{1}{2} \sigma_{x}\right)^{2} \frac{-1+2 v T+v^{2} T^{2}+e^{-2 v T}}{v^{2} T^{2}}+\frac{\sigma_{x}}{T} \int_{0}^{T} S^{2}(t) d t+ \\
+\left[\frac{1}{T} \int_{0}^{T} S^{2}(t) d t\right]^{2}+\frac{4 \sigma_{x}}{T^{2}} \int_{0}^{T} \int_{0}^{T} S(\tau) S(t) e^{v(\tau-1)} d t d \tau
\end{gathered}
$$




$$
P(J)=\frac{1}{\sqrt{4 \pi \Delta}} \exp \left(-\frac{(J-<J>)^{2}}{2 \Delta}\right)
$$

where $\Delta=\Delta\left(J_{S+x}\right)=\frac{4 \sigma_{x}}{T^{2}} \int_{0}^{T} \int_{0}^{T} S(\tau) S(t) e^{v(\tau-1)} d t d \tau$.

The processing of dependences (35), (36) allows one to determine the probability of the correct identification Ds of the regular component $\mathrm{s}(\mathrm{t})$ at the registration section.

To resolve the objective of identifying the causes of output variable value deviations from the forecast ones, we apply the method for determining the indirect measurement errors. If the output variable $y$ is functionally dependent on the output variables $x_{1}, x_{2}, \ldots, x_{h}$ with the known identification errors, the error $\Delta y$ is defined from the ratio

$$
(\Delta y)^{2}=\left(\frac{\partial f(x)}{\partial x_{1}} \Delta x_{1}\right)^{2}+\left(\frac{\partial f(x)}{\partial x_{2}} \Delta x_{2}\right)^{2}+\ldots+\left(\frac{\partial f(x)}{\partial x_{n}} \Delta x_{n}\right)^{2}
$$

For the considered problem the following deviations $\Delta y$ are known, the unknown deviations are $\Delta x$. The dependence (37) is also correct for any physical values available for observation. They may include not only the deviations $\Delta y$ and $\Delta x$ but also the derivatives, dispersions, etc. The problem can be recorded in the matrix form as follows

$$
\begin{aligned}
& W=\left[\begin{array}{c}
(\Delta Y)^{2} \\
(\Delta \dot{Y})^{2} \\
\left(\Delta S_{Y}^{2}\right)^{2}
\end{array}\right]_{;} \\
& A=\left[\begin{array}{c}
\left(\frac{\partial f(x)}{\partial x_{1}}\right)_{x_{\Delta}}^{2} \\
\vdots \\
\left(\frac{\partial f(x)}{\partial x_{n}}\right)_{x_{\Delta}}^{2}
\end{array}\right] \\
& B=\left[\begin{array}{c}
\left(\Delta x_{1}\right)^{2} \\
\vdots \\
\left(\Delta x_{n}\right)^{2}
\end{array}\right]_{;} \\
& W=A \times B
\end{aligned}
$$

At the solving of the system of equations set in the matrix form (41) there are several options that are possible. The number of unknown components can be higher, equal or less in relation to a number of equations. All these options are considered in detail within the linear algebraic theory [16]. In case the number of unknown components is larger than a number of equations, the system is statically indeterminate, in case they are equal, there is only one solution for the system, if the number is less, the system may have or not have any solutions. 


\section{Conclusion}

Therefore, the analysis of the set problems allows one to make the conclusion that, having the data on the dynamics of output variable change, it is always possible to identify the type of disturbance and change of the technical system and correct the treatment control as well as to calculate the time intervals after which one shall take into account some or other changes of the technological system.

\section{References}

1. Balakshin B.S. Basics of Mechanical Engineering Technology (M.: Mashinostroyeniye, 1969)

2. Bazrov B.M. Improving Precision of Part Manufacture By Means Of Their Treatment Control, Mashinostroitel, No. 3 P. 11-12 (1979)

3. Podurayev V.N. Technology of Physical and Mechanical Treatment Methods (M.: Mashinostroyeniye, 1988)

4. Mikhelkevich V.N. Grinding Automatic Control (M.: Mashinostroyeniye, 1975)

5. Yu.M. Solomentsev, V.G. Mitrofanov, K.V. Rybkin [et al.], Adaptive Control of Technological Processes (M.: Mashinostroyeniye, 1980_)

6. Petrakov Yu.V., Drachev O.I. Theory of Automatic Control of Technological Systems (M.: Mashinostroyeniye, 2009)

7. Pukhovsky Ye.S. Technological Basics of Flexible Automatic Production (Kiev: 1989)

8. A.A. Ignatyev, M. V.Vinogradov, V.V. Gorbunov [et al.], Monitoring of Machines and Grinding Processes in Bearing Manufacture (Saratov: SGTU, 2004)

9. L. Rokyta, Testing of grinding wheels for tools manufacturing. Annals of DAAAM for 2011 \& Proceedings of the 22nd International DAAAM Symposium, Vol. 22, No. 1 pp. 1137-1138, (2011)

10. F.V. Novikov, Theoretical analysis of the technological opportunities to reduce cutting temperature during grinding. Technologies of information's are in education, science and production, № 3, pp. 88-95, (2015)

11. S. Bratan, S. Roshchupkin, D. Revenko, Probabilistic Approach for Modeling Electroerosion Removal of Grinding Wheel Bond, Procedia Engineering V. 206, 14261431 (2017)

12. Yagyaev E.E. System of Diagnostics and Control of Grinding with Treatment Cycle Correction, Proceedings of Crimean Engineering and Pedagogical University. Issue 35. Technical Sciences. - Simferopol : NITs KIPU (SIC CEPU), 2012. P. 71-75.

13. Yagyaev E.E., Shron B.L., Ensuring stable quality of ground parts by monitoring the process system parameters, International Conference on Modern Trends in Manufacturing Technologies and Equipment (ICMTMTE 2017), MATEC Web of Conferences 129, 01069 (2017) https://doi.org/10.1051/matecconf/201712901069/ MATEC Web of Conferences is indexed in: Scopus, Google Scholar, EDP Sciences database.

14. Klyatskin V.I. Essays on Dynamics of Stochastic Systems, Russian Academy of Sciences, Institute of Atmosphere Physics n.a. A.M. Obukhov. M.: URSS, 2012. 442 p.

15. Dorf R., Bishop R. Modern Control Systems [translated from English by B.I. Kopylova] (M.: Laboratory of Basic Knowledge, 2002)

16. Umnov A.Ye. Analytical Geometry and Linear Algebra: Study Manual (3d edition, revised and enlarged - M.: MIPT, 2011) 